\title{
Fifteen Years of High Angular Resolution Studies of Mira's Atmosphere
}

\author{
Margarita Karovska \\ Harvard-Smithsonian Center for Astrophysics, 60 Garden Street, \\ Cambridge, MA 02138, USA
}

\begin{abstract}
.
I describe here results from high-angular resolution imaging studies of $o$ Ceti (Mira). In 1983, we discovered that the atmosphere of the prototype of Mira-type variables is not symmetric. Since then, a number of multiwavelength high-angular resolution observations have confirmed the presence of asymmetries in Mira's atmosphere, and detected asymmetries in the atmospheres of other Mira-type variables. The high-angular resolution images of Mira obtained over the past fifteen years, including recent HST observations, show that the strength and shape of the asymmetries change as a function of wavelength and time. Plausible mechanisms for these asymmetries include hot spots, nonspherical pulsations, interaction with the companion and bipolar outflow. The presence of asymmetries in Miras could have serious impact on evolutionary models, and on the development of model atmospheres.
\end{abstract}

\section{Introduction}

$o$ Ceti (Mira) is a pulsating cool giant (M2-M7 III) with a diameter of several hundred solar radii and a mass comparable to that of our Sun. The brightness of this prototype of long period large amplitude variable stars changes over seven magnitudes between its minimum and maximum, with a period of 332 days. Mira is losing mass at a rate of about $1 \times 10^{-7} \mathrm{M}_{\odot} / \mathrm{yr}$ (e.g. Bowers \& Knapp 1988) via a slow wind as it approaches the final stages of its evolution toward a planetary nebula. Mira has a companion at an angular distance of approximately 0.5 arcseconds. The companion is a white dwarf embedded in an accretion disk of variable luminosity.

Mira's system provides a unique laboratory for detailed study of the characteristics of pulsating giant atmospheres and wind accretion processes in interacting binaries, because it can be spatially resolved using high-angular resolution ground- and space-based observations, and the components can be studied individually.

\section{High Angular Resolution Observations}

Because of its large angular size, Mira can be easily resolved using high-angular resolution interferometric techniques. Pease (1931) first resolved this star using 
Michelson interferometry and measured a diameter of 47 mas at $575 \mathrm{~nm}$. Half a century later, Labeyrie et al. (1977) obtained the first high precision measurements of the diameter of Mira using the $5 \mathrm{~m}$ Palomar telescope and a new technique - speckle interferometry. Diameter measurements were made assuming spherical symmetry for the object. The speckle observations revealed a huge extension of Mira's atmosphere at optical wavelengths, from 50 mas to more than 100 mas. Measured diameters vary by factors as large as 2.5 over a few neighboring pseudo-continuum regions (Bonneau et al. 1982). These variations have been interpreted, quite roughly, in terms of optical depth variations due to the effects of pulsation on the structure of the extended atmosphere.

In November 1983 we carried out the first high-angular resolution imaging observations of this source using the Harvard-Smithsonian Astrophysical Observatory (CfA) speckle interferometry system. The observations were obtained at Mira's light curve phase 0.43 (Mattei, private communication) at the Steward Observatory $2.25 \mathrm{~m}$ telescope. We discovered a substantial asymmetry in Mira's images processed with Knox-Thompson image reconstruction techniques. The direction of the major axis of this asymmetry was nearly perpendicular to the direction toward the companion.

Follow up observations, carried out using larger aperture telescopes and therefore higher diffraction limited resolution, confirmed the presence of asymmetries in Mira's atmosphere. We carried out high-angular resolution speckle observations of Mira at several epochs using the 4-m telescopes at Kitt Peak and Cerro Tololo, and observed asymmetries at different wavelengths. For example, the observations carried out in 1988 at Cerro Tololo show an elongation in the $533 \mathrm{~nm}$ image with a position angle of the major axis of $135 \pm 10$ degrees, which is similar to the position angle of the asymmetry in the $775 \mathrm{~nm}$ image obtained at Kitt Peak one year earlier. However, the 1988 image shows some change in the geometry, and the degree of asymmetry does appear somewhat smaller. Since then, a number of observations using high-angular resolution techniques detected asymmetries in Mira and in other Mira-type variables using groundand space-based observations (e.g. Karovska et al. 1991; Haniff et al. 1992).

Recently, we imaged the individual components of Mira's binary system using the HST Faint Object Camera (FOC) (Fig. 1). The multiwavelength images combined with the objective prism spectra provide a unique perspective of this accreting system and its components at wavelengths ranging from 150 $\mathrm{nm}$ to $550 \mathrm{~nm}$ (Karovska et al. 1997). We determined the spectral energy distribution of each component unambiguously at UV and optical wavelengths and obtained the first high spatial resolution images at UV wavelengths.

The high angular resolution of the COSTAR-corrected FOC f/96 camera, and the small pixel size provided an opportunity for exploring Mira's atmosphere at minimum light and at spatial scales as small as 30 milliarcseconds. The images resolved Mira at several optical wavelengths, and for the first time in the UV (see Fig. 2 and Fig. 3). The size of Mira's atmosphere appears smaller in the quasi-continuum spectral regions than in the regions containing $\mathrm{TiO}$ bands. If this is interpreted in terms of a stratification in the atmosphere of Mira, then depending on the spectral region in which the observations were made, we effectively probed different layers in the atmosphere. 

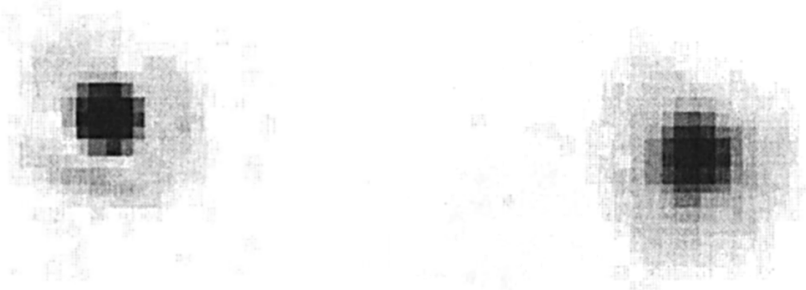

Figure 1. Direct HST/FOC images of Mira A (right) and Mira B (left) in the F501N filter. The separation between the components is 0.58 arcseconds.

The most surprising result came from the first UV image of Mira recorded using a filter centered at $346 \mathrm{~nm}$ (Fig. 2). The deconvolved image shows a hook-like appendage extending from Mira in the direction of the companion (Karovska et al 1997; NASA and STScI Press release STScI-PRC97-26, 1997). This feature could be material from the giant being gravitationally drawn toward the accreting companion, a physical phenomenon never directly observed at this small spatial scale.

We detected asymmetries in the Mira multiwavelength images deconvolved using the images of the companion as point sources. For example, the $501 \mathrm{~nm}$ deconvolved image of Mira shows an asymmetry in the extended atmosphere in the south-east direction (Fig. 3). The apparent size of the major axis of the dominant asymmetry as measured using elliptical gaussian fitting to the deconvolved images is $56+/-1$ mas, with a position angle of $175+/-2$ degrees. The estimated ratio between the minor and the major axis of the asymmetry is 0.2 .

This asymmetry is similar to the asymmetries previously detected using ground-based interferometry (e. g. Karovska et al. 1991; Haniff et al. 1992). HST multiwavelength images also detected low brightness arc-like features in Mira's circumstellar environment. These structures are reminiscent of the morphology of pre-planetary nebulae and appear to carry signatures of a bipolar outflow.

\section{Discussion}

High-angular resolution observations of Mira show direct evidence that the atmosphere of this star departs substantially from spherical symmetry. The appearance of the asymmetry changes as a function of time and wavelength.

The cause of the asymmetries in the Mira atmosphere has not yet been determined. Thermal, density, or dynamic instabilities could create asymmetries in brightness distribution on the surface of Mira, or geometric asymmetries with 


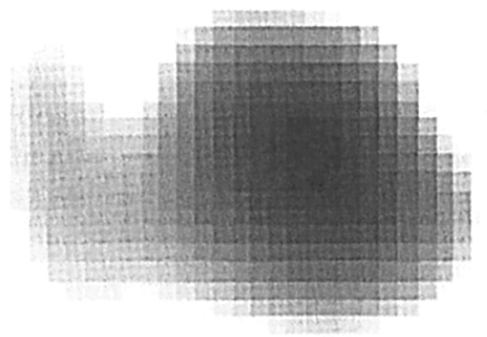

Figure 2. The first UV image of Mira showing a hook-like appendage extending from Mira in the direction of the companion (NASA and STScI Press release STScI-PRC97-26, 1997). This deconvolved image has been magnified for visualization purpose.

orientation that changes with time. For example, dynamic instabilities apparent in some atmospheric models can produce "shock pairing" which in turn could produce departures from spherical symmetry (Willson \& Bowen 1988). Nonradial pulsations could also create departures from spherical symmetry similar to the asymmetries observed in Mira. Convective instabilities (Schwarzschild 1975) could result in a formation of large supergranules or spots on the photosphere which if partially resolved could create asymmetries in the diffraction limited images.

Rapid rotation, or the companion and its orbital motion, could affect the upper atmosphere of Mira and create favorable conditions for a bipolar outflow. In fact, the HST images show structures that could be associated with a bipolar outflow. This is in agreement with radio observations which detected an extended bipolar outflow in the CO $\mathrm{J}=2-1$ and $\mathrm{J}=1-0$ emission of the circumstellar envelope of Mira (Planesas et al. 1990). The observed structure has been interpreted as partial collimation of the stellar wind due to an enhanced density of gas in the equatorial plane of the inner envelope of Mira.

The presence of substantial asymmetries in the atmospheres of Mira-type variables has an important effect on the measured quantities and the model atmospheres for these stars. For example, even without the asymmetries, it is difficult to determine directly the "true" photospheric diameter, the crucial parameter for establishing the effective temperature and the mode of pulsation because of the effects of the extended atmosphere (Willson \& Bowen 1988). Currently, observers use model brightness distribution derived from various model atmosphere codes to correct the measured diameters and estimate the "photospheric" diameter and the mode of pulsation of Miras. These models are often very simplistic, and assume that the extended Mira atmosphere is spherically 


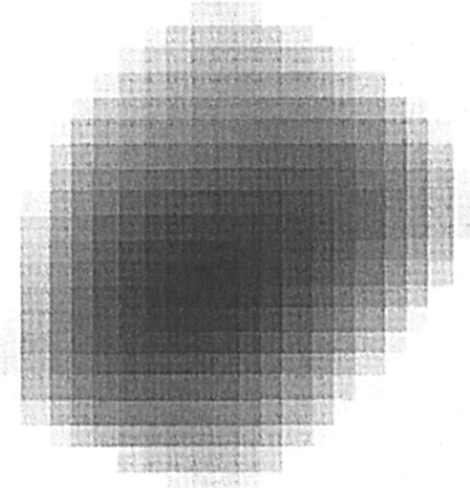

Figure 3. Deconvolved HST image of Mira at $501 \mathrm{~nm}$ showing substantial asymmetry in the south-east direction. The image has been magnified five times using bicubic spline interpolation for visualization purpose.

symmetric. Therefore, the results obtained using these models should be handled with care.

Continuing the high-angular resolution studies of the detailed structure of Mira's atmosphere, and of the origin and the nature of the asymmetries is crucial for further development and testing of model atmospheres and evolutionary models.

Acknowledgments. This work was supported in part by STScI grant GO5822.01-94A to the Smithsonian Astrophysical Observatory. MK is a member of the AXAF Science Center and is partially supported by NASA, Contract \#NAS8-39073.

\section{References}

Bonneau D., Foy R., Blazit A., Labeyrie A., 1982, A\&A 106, 235

Haniff C.A, Ghez A.M., Gorham P.W., et al., 1992 AJ 103, 1667

Karovska M., Nisenson P., Papaliolios C., Boyle R.P., 1991, ApJ 374, L51

Karovska M., Hack W., Raymond J., Guinan E., 1997, ApJ 482, L175

Labeyrie A., Koechlin L., Bonneau D., Blazit A., Foy R., 1977, ApJ 218, L75

Pease F.G., 1931, Ergebn. Exacten. Naturwis. 10, 84

Planesas P., Kenney J.D.P., Bachiller R., 1990, ApJ 364, L9

Schwarzschild M., 1975, ApJ 195, 137

Wilson R.W., Baldwin J.E., Busher D.F., Warner P.J., 1992, MNRAS 257, 369 


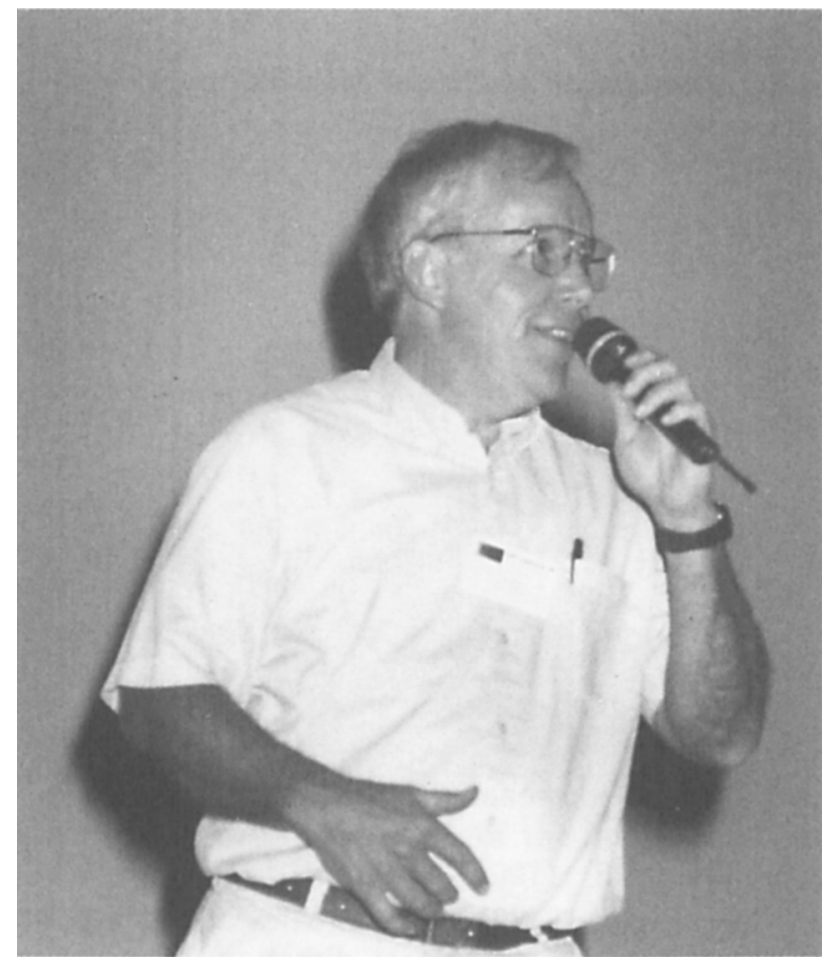

Peter Wood and Paola Marigo during the pannel discussion: "Stellar evolution on the AGB: a critical view on the models"

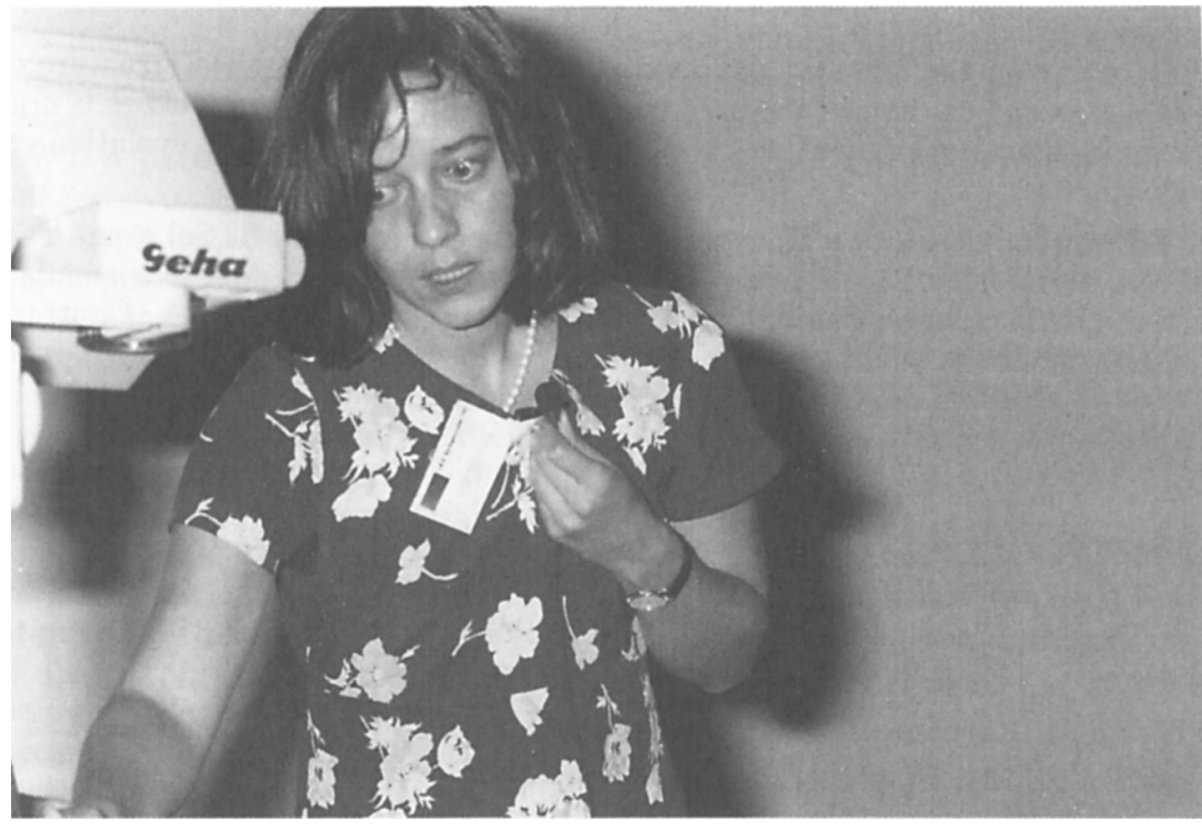

